\section{IN BRIEF}

\section{$\Rightarrow$ SJÖGREN SYNDROME}

\begin{abstract}
Abatacept no better than placebo for pSS
In the single-centre Abatacept Sjögren Active Patients phase III (ASAP-III) study, no difference was seen in disease activity (as measured by ESSDAl) in patients with primary Sjögren syndrome $(p S S)$ treated with abatacept $(n=40)$ compared with those who received placebo $(n=39)$ after 24 weeks. Patient-reported symptoms (as measured by ESSPRI) also did not differ between the groups, although $\lg \mathrm{G}$ and rheumatoid factor concentrations were decreased in the abatacept group at 24 weeks. Further studies are needed to evaluate the effects of abatacept on specific systemic manifestations of pSS.

ORIGINAL ARTICLE van Nimwegen, J. F. et al. Abatacept treatment for patients with early active primary Sjögren's syndrome: a single-centre, randomised, double-blind, placebocontrolled, phase 3 trial (ASAP-III study). Lancet Rheumatol. https://doi.org/10.1016/ S2665-9913(19)30160-2 (2020)
\end{abstract}

\section{FIBROINFLAMMATORY DISORDERS}

\section{Leflunomide plus glucocorticoids for IgG4-RD}

In a 12-month, open-label, randomized, controlled trial, combination therapy with leflunomide and glucocorticoids was superior to glucocorticoid monotherapy in preventing relapse of $\lg$ G4-related disease (lgG4-RD). Patients who received the combination therapy $(n=33)$ also had a shorter time to complete response and a longer duration of remission than those who received glucocorticoid monotherapy $(n=33)$. The combination therapy resulted in a lower cumulative dosage of glucocorticoids, suggesting that leflunomide could be used as a steroid-sparing agent in the management of lgG4-RD.

ORIGINAL ARTICLE Wang, Y. et al. Additive effect of leflunomide and glucocorticoids compared with glucocorticoids monotherapy in preventing relapse of lgG4-related disease: a randomized clinical trial. Semin. Arthritis Rheum. https://doi.org/10.1016/ j.semarthrit.2020.01.010(2020)

\section{PAIN}

\section{Tofacitinib alleviates pain in RA, PsA and AS}

Post-hoc analysis of data from 3,330 patients who received $5 \mathrm{mg}$ or $10 \mathrm{mg}$ tofacitinib twice daily for the treatment of rheumatoid arthritis (RA), psoriatic arthritis (PsA) or ankylosing spondylitis (AS) in phase II and III studies reveals that tofacitinib treatment was associated with rapid and sustained alleviation of pain. Overall, improvements were seen in all pain assessments (including unidimensional pain measures and individual pain components of multidimensional measures) with tofacitinib treatment compared with placebo. Tofacitinib reduced pain at both dosages and regardless of inadequate response to previous treatments.

ORIGINAL ARTICLE Ogdie, A. et al. Efficacy of tofacitinib in reducing pain in patients with rheumatoid arthritis, psoriatic arthritis or ankylosing spondylitis. RMD Open 6 , e001042 (2020)

\section{$\Rightarrow$ GOUT}

\section{$A B C G 2$ SNP associated with early-onset gout}

Among 10 single nucleotide polymorphisms (SNPs) strongly associated with serum urate concentrations, only ABCG2 rs2231142 was associated with early-onset gout (defined as symptom onset before 40 years of age). The association was present in people in New Zealand of European and Polynesian ancestry (in the Genetics of Gout in Aotearoa study) and was confirmed in two replication cohorts. Early-onset gout was associated with an increased frequency of flares.

ORIGINAL ARTICLE Zaidi, F. et al. Systematic genetic analysis of early-onset gout: ABCG2 is the only associated locus. Rheumatology https://doi.org/10.1093/rheumatology/kez685 (2020)

\title{
Epigenetic imprinting maintains profibrotic phenotype in SSc
}

Aberrant activation of fibroblasts underlies the pathogenesis of fibrotic diseases such systemic sclerosis (SSc). New findings published in The Journal of Clinical Investigation support the contribution of epigenetic imprinting for maintaining the persistently activated pathological phenotype of fibroblasts in SSc.

"In this study, we link two core mechanisms of fibrosis: epigenetics and TGF $\beta$-STAT3 signalling," reports corresponding author Jörg Distler. Suppressor of cytokine signalling 3 (SOCS3) is a negative regulator of TGF $\beta$-STAT3 signalling that is normally induced as part of a negative-feedback response. "We demonstrate that persistent activation of TGF $\beta$ signalling induces the expression of DNA methyltransferases DNMT3A and DNMT1 to silence the expression of SOCS3," explains Distler. "This mechanism further amplifies the stimulatory effects of TGF $\beta$ on STAT3 signalling and amplifies TGF $\beta$-induced fibroblast activation and tissue fibrosis."

Indeed, the expression of SOCS3 was downregulated in the skin and in skin fibroblasts of patients with SSc compared with that of healthy individuals, and expression was also downregulated in the skin of mice with bleomycin-induced fibrosis or fibrosis caused by constitutive activation of TGF $\beta$ receptor type I (TBR $1^{\text {act }}$ mice). Treatment of fibroblasts from healthy individuals with the DNMT inhibitor 5 -azacitidine (5-aza) prevented TGF $\beta$-mediated repression of SOCS3 expression following prolonged stimulation in vitro.

Methylation-specific PCR and methylated DNA immunoprecipitation analysis suggested that TGF $\beta$ signalling induces the expression of DNMT3A and DNMT1 via the canonical TGF $\beta-S M A D$ pathway to promote hypermethylation of the SOCS3 promoter and silence SOCS3 expression.

Manipulating the expression of SOCS3 in fibroblasts in vitro using siRNA-mediated knockdown, incubation with 5-aza or forced overexpression of SOCS3 showed that SOCS3 limits TGF $\beta$-dependent fibroblast activation (as measured by TGF $\beta$-mediated expression of COL1A1 and COL12A2 mRNA, expression of $\alpha$-smooth muscle actin, release of collagen protein and formation of stress fibres).

Notably, in vitro and in vivo findings confirmed that SOCS3 inhibits JAK2-STAT3 signalling, and that 5 -aza can restore SOCS3-mediated regulation of JAK-STAT signalling.

Fibroblast-specific deletion of SOCS3 in mice exacerbated bleomycin-induced or TBR $1^{\text {act }}$ induced skin fibrosis. By contrast, treatment with 5-aza or fibroblastspecific deletion of DNMT3A ameliorated fibrosis in both models. Notably, 5-aza treatment not only prevented fibrosis, but also induced regression of pre-established bleomycin-induced skin fibrosis.

"We are currently evaluating options to transfer these findings from bench to bedside and are discussing options to run a clinical trial with DNMT inhibitors such as 5-aza," says Distler.

Jessica McHugh

ORIGINAL ARTICLE Dees, C. et al. TGF $\beta$-induced epigenetic deregulation of SOCS3 facilitates STAT3-signaling to promote fibrosis. J. Clin. Invest. https://doi.org/10.1172/JCI122462 (2020)

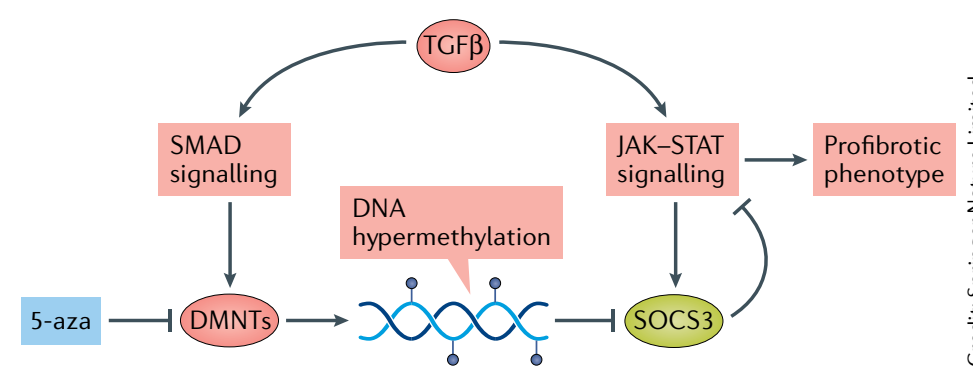

